$\underline{\text { Review Article }}$

\title{
ANTIVIRAL DRUGS FOR INFLUENZA VIRUSES
}

\section{S. K. MANIRUL HAQUE, MOHAMED ELZAGHEID*}

Department of Chemical and Process Engineering Technology, Jubail Industrial College, P. 0. Box No. 10099, Zip Code 31961, Jubail Industrial City, Saudi Arabia

Email: elzagheid_m@jic.edu.sa

Received: 13 Jan 2019 Revised and Accepted: 29 Mar 2019

\begin{abstract}
Antiviral drugs have significant action against influenza viruses A and B. Virus spread deadly disease in which many people die, and the country economy greatly suffer. Presently, most of the people need to get vaccination, which is depending on the dose limit in humans. It reacts directly or sometimes indirectly in the form of metabolites. However, it is mandatory to know how much drug is absorbed or metabolites concentration after administered. Therefore, pharmacokinetics data is very crucial for all drugs. Our review discusses the mechanism of drugs action and their activity and also describes how antiviral drugs and their metabolites are determined using highly sensitive instruments such as high-performance liquid chromatography (HPLC), ultra-pressure liquid chromatography (UPLC), and mass spectrometry (MS). Therefore, the present review gives brief information about antiviral drugs, their activity, biotransformation and analytical methods for quantification and this information will be helpful for any future studies done by experts in this field and will be beneficial for research scientists and influenza experts of all over the globe.
\end{abstract}

Keywords: Antiviral drugs, Influenza virus, Oseltamivir, Laninamivir, Zanamivir, Peramivir, Metabolite, HPLC, UPLC, LCMS

(C) 2019 The Authors. Published by Innovare Academic Sciences Pvt Ltd. This is an open-access article under the CC BY license (http://creativecommons.org/licenses/by/4.0/) DOI: http://dx.doi.org/10.22159/ijpps.2019v11i5.32024

\section{INTRODUCTION}

Respiratory system is commonly infected with influenza viruses [1] and mainly orthomyxoviridae family (A, B, C). Worldwide, 2.5-5 hundred thousand people were reported death and 3-5 million diagnosed with acute illness [2] annually. Viruses classified with respect to activity of glycoproteins neuraminidase (N/NA), hemagglutinin $(\mathrm{H} / \mathrm{HA})$ and $\mathrm{M} 2$ ion channel. Influenza $\mathrm{A}$ and $\mathrm{B}$ are seasonal influenza with different subtypes such as H1-H16 (16 hemagglutinin) and N1-N9 (9 neuraminidase). Well-known viruses $\mathrm{H} 1 \mathrm{~N} 1$ and $\mathrm{H} 3 \mathrm{~N} 2$ are generally subtyped of seasonal virus (Influenza A). The avian influenza virus H5N1 [3-5] have great interest among the people but swine influenza (novel H1N1) is completely different from seasonal H1N1 influenza virus [6]. In the 20 th century, 20-50 million people from different countries were killed with Spanish flu, 1-2 million from Asian flu, and 7 hundred thousand from Hong Kong flu $[7,8]$. Virus infection was initiated by hemagglutinin after binding with a receptor called Sialic acid, 1 (fig. 1). Then other cells infected by new viruses with the help of neuraminidase $[9,10]$. Life threat influenza can only be overcome with annual immunization. However, the limitation related to efficacy, timeline and design of the vaccine production increase the need for antiviral drugs. The approved antiviral drugs M2 ion channel and NA inhibitors are the basic option for therapeutic treatment with prevention. This classification based on resistance behavior, tolerance study, pharmacokinetics and drugs mechanism of action [11-17].

\section{Neuraminidase inhibitors}

Proteins ( $\mathrm{H}$ and $\mathrm{N}$ ) are available on the walls of influenza virus surfaces. New viral molecule was released by host cell due to enzymatic function of neuraminidase, will help to catalyzed glycoside hydrolysis. As a result, $\alpha$-ketosidically connected sialic acid with glycolipid, glycoprotein, and hemagglutinin divided increased infectious molecule. However, the nascent virus is still securing with host cell due to an interaction between HA/HA receptor. The viral molecule exhibit HA/HA receptors on the surface, which enables new molecules stick together. The viral particles emerged from the clump and host cell when neuraminidase receptor removed sialic acid. In the respiratory, glycosylated components are digested by neuraminidase, contribute viral infection. Therefore, design-based modern drug act on the site of neuraminidase inhibitors, depend on its clinical efficacy, mechanism, genetic role are the key feature for success [18-20]. The neuraminidase inhibitors prompt action and excellent mortality is the main reason for its demand [21, 22]. The drugs and their pharmaceutical formulations worked for the treatment of deadly disease are Oseltamivir 2, Laninamivir 3, Zanamivir 4, Peramivir 5 [23-25] (fig. 1), established on sialic acid transformation [26-28]. Influenza experts from different countries set up a neuraminidase inhibitors susceptibility network (NISN) for the outcome of clinical result and monitoring development process. This committee is participating to monitor liability studies for encouraging the people to know the exact method of evaluation and investigation process to control influenza virus.<smiles>CC(=O)N[C@H]1[C@H](O)C[C@@](O)(C(=O)O)O[C@H]1[C@H](O)[C@H](O)CO</smiles><smiles>CCOC(=O)C1=C[C@@H](OC(CC)CC)[C@H](NC(C)=O)[C@H](N)C1</smiles>

Oseltamivir (2)<smiles>CO[C@@H]([C@H]1OC(C(=O)O)=C[C@H](NC(=N)N)[C@H]1NC(C)=O)[C@H](O)CO</smiles><smiles>CC(=O)N[C@H]1[C@H](NC(=N)N)C=C(C(=O)O)O[C@H]1[C@H](O)[C@H](O)CO</smiles>

Zanamivir (4)<smiles>CCC(CC)C(NC(C)=O)[C@@H]1C(NC(=N)N)C[C@H](C(=O)O)[C@@H]1O</smiles>

Peramivir (5)
Fig. 1: Chemical structures of Sialic acid 1, Oseltamivir 2, Laninamivir 3, Zanamivir 4, and Peramivir 5 


\section{M2 ion channel inhibitors}

Amantadine 6 and Rimantadine 7 (fig. 2) licensed by United States of America (USA) in the years 1966 and 1993 respectively for the treatment of influenza A as M2 ion inhibitor. Allosteric inhibition controls the movement and activity of M2 by the presence of methyl group in amantadine; consequently, it blocks the release of RNA. It was found that M2 ion is successful for influenza A but ineffective towards influenza B. Therefore, these drugs are precise only for influenza A. M2 inhibitor has extraordinary response to antibiotic resistance $[29,30]$, but it is very less compared to neuraminidase inhibitors [31, 32]. Through endocytosis process, host cell allows viruses to enter. At low $\mathrm{pH}, \mathrm{M} 2$ channel permits the virus to viral interior due to an activity of endosome responsible for maturation and growth of molecule. The reproduction is continued in cytoplasm, viral ribonucleic acid (RNA) and genetic material released from protein matrix [33]. Therefore, it is very important to develop inhibitors functioning against the virus but in native condition.

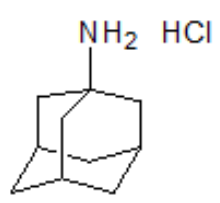

Amantadine $\mathrm{HCl}(6)$

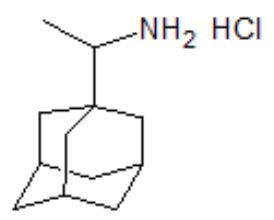

Rimantadine $\mathrm{HCl}(7)$
Fig. 2: Chemical structures of Amantadine 6 and Rimantadine 7

\section{Biotransformation}

\section{Oseltamivir}

Oseltamivir phosphate 8 is significant ester prodrug, applied orally for prevention and curing of influenza disease (A and B). It's brand name Tamiflu is available in quantity of $75 \mathrm{mg}$. The approved regimen for adults is $75 \mathrm{mg}$ daily two times for $5 \mathrm{~d}$ with respect to influenza ( $A$ and $B$ ), but it is different for the prophylactic regimen. For control and prevention, daily dose is $75 \mathrm{mg}$ once; for a minimum of $10 \mathrm{~d}$ up to $42 \mathrm{~d}$. The intake dose for healthy participants follows linear pharmacokinetics in the range of 75-675 mg [34-36]. Oseltamivir is absorbed in the gastrointestinal tract then metabolized and converted into Oseltamivir carboxylate 9 (fig. 3) in the liver and later on, uniformly distributed in the body. After oral administration, therapeutic action continues for $30 \mathrm{~min}$ with the metabolite ( $80 \%$ ) but the remaining $20 \%$ available $3-4 \mathrm{~h}$. in plasma. The drug half-life is $1.8 \mathrm{~h}$. It was found that renal disease may affect active metabolite clearance and found that cytochrome P450 has only $3 \%$ interaction with plasma protein. Similar action occurs during glucuronosyl transferases [37-38].

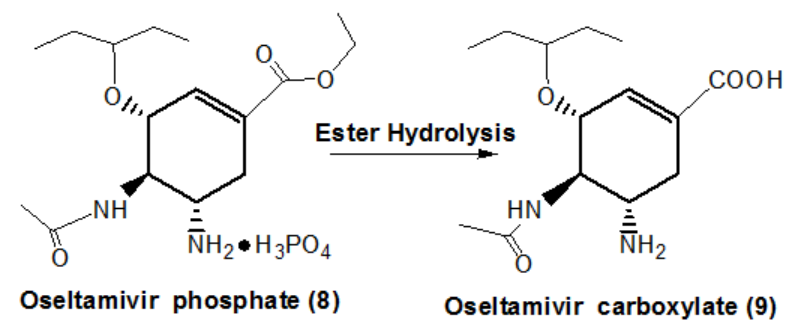

Fig. 3: Chemical structures of Oseltamivir phosphate 8 (prodrug) and Oseltamivir carboxylate 9 (active metabolite)

\section{Zanamivir}

Relenza is the trade name of Zanamivir, an anti-influenza inhibitor, which obtained the regulatory approval in 1999. It targets respiratory site and available as inhaled powder. The pharmaceutical formulation has high potential against people diagnosed with deadly disease influenza A (H5N1). The maximum amount of $20 \mathrm{mg}$ (10 mg twice) is allowed daily as inhalation, continued for 5 consecutive days. However, for prophylaxis, time and quantity limit is $10 \mathrm{mg}$ daily for $28 \mathrm{~d}$ for children age five or more, and same for adults [39-40]. The nasal spray set down into posterior nasal and nostril approximately $88 \%$ but $13 \%$ in lung, $78 \%$ in oropharynx deposited active site for replication of influenza virus [41]. Settle down in stomach (17\%) and lung (2\%) dose intake orally after fifty minutes [42]. Sputum and nasal wash sample resulted concentration about 50\% inhibitory after 24 and $12 \mathrm{~h}$. respectively [43] for healthy volunteers with $10 \mathrm{mg}$ daily dose but $20 \mathrm{mg}$ amount with infected sample quantified after $4 \mathrm{~d}$ [44]. The unchanged drug (90\%) was found with excreted urine sample recommended without biotransformation [41]. Therefore, the drug not metabolized in the liver.

\section{Peramivir}

Peramivir is a predominant cyclopentane based neuraminidase inhibitor. The binding capacity of the inhibitor will be half (IC50) with a concentration in the range $0.09-1.39 \mathrm{nM}$ and $0.6-10.8 \mathrm{nM}$ respectively for influenza A and B strains. This value is for Oseltamivir carboxylate 0.01-2.24 $\mathrm{nM}, 6.39-24.3 \mathrm{nM}$ and for Zanamivir $0.30-2.32 \mathrm{nM}, 1.53-17.0 \mathrm{nM}$ [45]. The drug is productive without any complication for seasonal influenza viruses [46]. The Peramivir IV was first launched with trade name Rapiacta in Japan (2010). The drug prescribed for acute treatment in adults is $300 \mathrm{mg}$ daily but for severe cases, hospitalized patients, $600 \mathrm{mg}$ daily dose is recommended. The drug eliminated through renal and not metabolized in the human liver.

\section{Laninamivir}

The function of Laninamivir 3 is similar to Zanamivir, potent neuraminidase inhibitor for influenza viruses (A and B) [47-48]. Laninamivir octanoate is an inactive prodrug and the commercially approved product (inavir) is available in Japan since 2010 [49]. Single dose of Laninamivir octanoate hydrate is limited to children with age above $10 \mathrm{y}$ is $40 \mathrm{mg}$ and below $10 \mathrm{y}$ is $20 \mathrm{mg}$. Laninamivir, active metabolite of Laninamivir octanoate (LO) formed within $24 \mathrm{~h}$ due to hydrolysis in the octanoyl ester site. When LO is mixed with water in the human body, equilibrated to form 3-acyl form 10, 2-acyl form 11 (fig. 4) known as major and minor metabolites [50].<smiles>CCCCCCCC(=O)OC[C@H](O)[C@H](OC)[C@@H]1OC(C(=O)O)=C[C@H](NC(=N)N)[C@H]1NC(C)=O</smiles>

Laninamivir octanoate (10), 3-acyl form (major)<smiles>CCCCCCCC(=O)O[C@H](CO)[C@@H](OC)[C@@H]1OC(C(=O)O)=C[C@H](NC(=N)N)[C@H]1NC(C)=O</smiles>

Laninamivir octanoate (11), 2-acyl form (minor)

Fig. 4: Chemical structures of Laninamivir octanoate 3-acyl form 10 and 2-acyl form 11 


\section{Amantadine}

Amantadine 6 is a very important drug as M2 proton channel inhibitor for influenza A. Symmetrel is the brand name normally used for amantadine. Its rate of absorption is high for young people compared to elder independents. Renal insufficiency does not appear to affect absorption, nor does the formulation used. First relative bioavailability studies of the oral administration reported that $86 \%$ recovery of the original dose $(2-4 \mathrm{mg} / \mathrm{kg})$ within $96 \mathrm{~h}$ from urine samples of five healthy men [51]. In case of ingestion after $72 \mathrm{~h}, 105 \mathrm{mg}$ (52.5\%) of the original dose was found in old man (84 y) urine [52]. Eight metabolites originated during metabolism. Besides the major metabolite produced by $\mathrm{N}$-acetylation which is $\mathrm{N}-$ acetylamantadine 12; other metabolites were observed. These are $\mathrm{N}$-methylamantadine $13, \mathrm{~N}$-dimethylamantadine $14, \mathrm{~N}-$ methyleneamantadine $15, \mathrm{~N}$-formylamantadine 16 , and metabolites 17, 18 and 19 (fig. 5) [53].

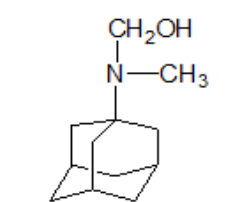

(17)

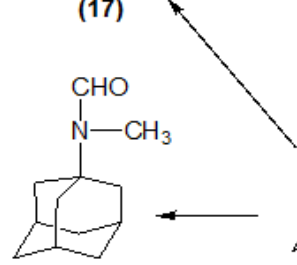

(18)

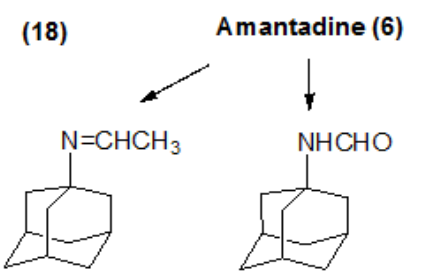

(16)

9)

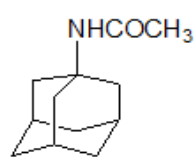

(12)
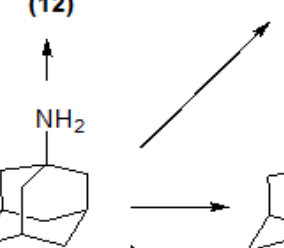

(13) res of amanta metabolites

\section{Rimantadine}

Pharmaceutical formulation of Rimantadine is known as flumadine. Recommended daily dose for adults is $200 \mathrm{mg}$ for $11-42 \mathrm{~d}$, for children of 1-9 y (5 mg/kg) $150 \mathrm{mg}$ is the maximum limit. Several studies concluded that $10 \%$ of the amantadine dose and $75 \%$ of the Rimantadine dose are metabolized in the liver [54-57]. The metabolites are determined as free Rimantadine 7, mhydroxyrimantadine 20, p-hydroxyrimantadine (equatorial 21 and axial 22) (fig. 6) [58-62].

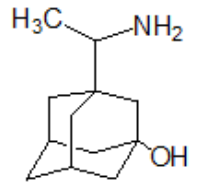

(20)

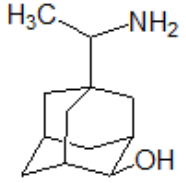

(21)

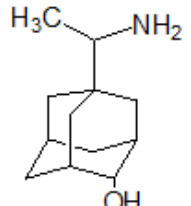

(22)
Fig. 6: Chemical structures of $m$-hydroxyrimantadine (20) and the equatorial (21) and the axial (22) epimers of $p$ hydroxyrimantadine

\section{Analytical techniques \\ UV-visible spectrophotometer}

UV-visible spectrophotometer is a simple and common technique for quantification of pure, pharmaceutical formulations and biological fluids. First order derivative spectrophotometry applied for Oseltamivir capsules having sophisticated additional software for pharmaceutical industry [63]. The drug $(\lambda \max =217,208.5 \mathrm{~nm})$ is stable and suitable with different stress condition in dosage form $[64,65]$. Potassium permanganate as oxidizing agent in alkaline medium, no interference from excipients for capsules [66]. The pure drug with formulation is determined by monitoring reaction mechanism in buffer medium with 4-chloro-7-nitrobenzo-2-oxa-1, 3-diazole [67]. Zanamivir is evaluated in tablet formulation at $260 \mathrm{~nm}$, validated respect to an international conference on harmonization guidelines [68,69]. Amantadine derivative and ion pair complex was developed with 1, 2-naphtoquinone-4-sulfonate, bromocressol green (BCG), bromophenol blue (BPB), bromothymol blue (BTB) and methyl orange in pharmaceutical preparation and plasma samples [70-73]. Rimantadine can also be determined spectrophotometrically using 1 , 2-naphtoquinone-4-sulfonic acid sodium salt as derivatizing reagent in pharmaceutical dosage form [74].

\section{High-performance liquid chromatography}

High-performance liquid chromatography (HPLC) is an accurate instrument for pharmaceutical analysis. The inhibitor demand is increasing with time in the world because influenza viruses causes significant health alert. All determined drugs either applying ultraviolet detector or fluorescence detector. As well as used $\mathrm{C}_{18}$ and $\mathrm{C}_{8}$ column with a different manufacturer. The single mobile phase is usually a combination of two or more solvents. The ratio of the mobile phase is changed with time; gradient method investigation resulted in drugs with a short time. During the analysis buffer also added for the preparation of the mobile phase. Therefore, for the quantification of neuraminidase and M2 ion channel inhibitor, numerous HPLC methods are developed based on the mobile phase composition and the stationary phase for pure bulk drug, tablet dosage form and biological fluids (table 1) [75-102].

\section{High-performance thin layer chromatography}

Currently, the high-performance thin layer chromatography (HPTLC) has high demand because it involves fewer samples cleanup, high throughput value, low-cost methodology. The fundamental advantage of HPTLC leads to compute several samples at one time. Oseltamivir study performed on silica gel 60F-254 coated aluminum plate consists of mobile phase (toluene: methanol: ammonia=3.5:1.5:0.2, v/v/v), detected at $R_{f}$ value $(0.45 \pm 0.02)$ for bulk and its dosage form [103]. This technique applied to evaluate Zanamivir in a pharmaceutical formulation having a correlation coefficient close to one $[90,104]$.

\section{Capillary zone electrophoresis}

Capillary zone electrophoresis (CZE) is strong enough to large molecules additionally for small (organic/inorganic), separation technique controlled by electric field inside narrow bore capillaries. The method has several benefits with respect to fewer amounts of solvent, analyte and high resolution, applicable to forensic, environmental, clinical and pharmaceutical industry. It is a specific technique for the separation of the molecule along with enantiomers. The generic version of Oseltamivir was separated on fused capillary under potential $(-15 \mathrm{kV})$ at room temperature. The analysis time is $1.5 \mathrm{~min}$ and detected at $226 \mathrm{~nm}$ included phosphate buffer [105]. Unmodified silica capillary, 4methylbenzylamine in ethanol $(20 \%)$ as background electrolyte, maintained $+20 \mathrm{~V}$ for separation at $210 \mathrm{~nm}$ within $3 \mathrm{~min}$ in Rimantadine tablets [106]. Pharmaceutical products can be estimated with derivatization agent 1, 2-naphthoquinone-4sulfonic acid by CZE in alkaline medium [107]. 
Table 1: Important parameters for the determination of drugs using HPLC

\begin{tabular}{|c|c|c|c|c|c|c|}
\hline Drug & Mobile phase & Stationary phase & $\begin{array}{l}\text { Flow rate } \\
(\mathrm{ml} / \mathrm{min})\end{array}$ & $\begin{array}{l}\text { Detector } \\
(\mathrm{nm})\end{array}$ & Application & Reference \\
\hline \multirow[t]{14}{*}{ Oseltamivir } & Acetonitrile: Methanol $=50: 30, \mathrm{v} / \mathrm{v}$ & $\begin{array}{l}\text { Purosphere } \mathrm{C}_{18} \\
(250 \times 4.6 \mathrm{~mm}, 5 \mu \mathrm{m})\end{array}$ & 1.0 & 227 & API & [75] \\
\hline & $\begin{array}{l}\text { Potassium dihydrogen } \\
\text { orthophosphate }(\mathrm{pH} 3.2) \text { : } \\
\text { Acetonitrile: Methanol = 60:20:20, } \\
\text { v/v/v }\end{array}$ & $\begin{array}{l}\text { YMC Pro } C_{8} \\
(150 \times 4.6 \mathrm{~mm}, 5 \mu \mathrm{m})\end{array}$ & 1.5 & 207 & Capsules & [76] \\
\hline & $\begin{array}{l}\text { Triethylamine (0.1\%): Methanol = } \\
60: 40, \mathrm{pH} 10.04 \text { with Conc } \mathrm{HCl}\end{array}$ & $\begin{array}{l}\text { Water's X Bridge } C_{18} \\
(250 \times 4.6 \mathrm{~mm}, 5 \mu \mathrm{m})\end{array}$ & 0.9 & 226 & $\begin{array}{l}\text { Bulk and } \\
\text { pharmaceutical } \\
\text { formulations }\end{array}$ & [77] \\
\hline & $\begin{array}{l}\text { Phosphate buffer }(0.02 \mathrm{M}, \mathrm{pH} 5 \text { with } \\
0.02 \text { M TEA): Methanol }=50: 50, \mathrm{v} / \mathrm{v}\end{array}$ & $\begin{array}{l}\text { Purosphere Star } \\
\text { RP-18e }(150 \times 4.6 \\
\mathrm{mm}, 5 \mu \mathrm{m})\end{array}$ & 1.5 & 215 & Bulk drug and capsules & [78] \\
\hline & $\begin{array}{l}\text { Mobile phase } \mathrm{A}-\mathrm{KH}_{2} \mathrm{PO}_{4} \text { buffer }(0.05 \\
\mathrm{m}, \mathrm{pH} 6): \text { Acetonitrile }=90: 10, \mathrm{v} / \mathrm{v} \\
\text { Mobile phase } \mathrm{B}-\mathrm{KH}_{2} \mathrm{PO}_{4} \text { buffer }(0.05 \\
\text { m, pH 6): Acetonitrile }=10: 90, \mathrm{v} / \mathrm{v} \\
\text { Gradient Programme }\end{array}$ & $\begin{array}{l}\text { ODS }(50 \times 4.6 \mathrm{~mm} \text {, } \\
5 \mu \mathrm{m})\end{array}$ & 1.5 & 220 & Bulk drug & [79] \\
\hline & $\begin{array}{l}\text { Ammonium acetate buffer }(\mathrm{pH} 6.9) \text { : } \\
\text { Acetonitrile }=60: 40, \mathrm{v} / \mathrm{v}\end{array}$ & $\begin{array}{l}\text { Purosphere } \mathrm{C}_{18} \\
(250 \times 4.6 \mathrm{~mm}, 5 \mu \mathrm{m})\end{array}$ & 1.0 & 220 & Bulk drug and dosage & {$[80]$} \\
\hline & $\begin{array}{l}\text { Sodium acetate buffer }(\mathrm{pH} 4.5) \text { : } \\
\text { Acetonitrile }=55: 45, \mathrm{v} / \mathrm{v}\end{array}$ & $\begin{array}{l}\text { Phenomenex Luna } \\
\mathrm{C}_{18}(250 \times 5 \mathrm{~mm}, 5 \\
\mathrm{mm})\end{array}$ & 1.5 & 231 & Bulk and dosage form & {$[81]$} \\
\hline & $\begin{array}{l}\text { Acetonitrile: Nitric acid }(10 \mathrm{mmol} \text {, } \\
\mathrm{pH} 3)=60: 40, \mathrm{v} / \mathrm{v}\end{array}$ & $\begin{array}{l}\text { Phenomenex } \mathrm{C}_{18} \\
(250 \times 4.6 \mathrm{~mm}, 5 \mu \mathrm{m})\end{array}$ & 1.0 & $\begin{array}{l}\lambda \text { ex } 470 \\
\lambda \text { em } 541\end{array}$ & $\begin{array}{l}\text { Capsules and spiked } \\
\text { plasma }\end{array}$ & {$[82]$} \\
\hline & $\begin{array}{l}\text { Potassium dihydrogen } \\
\text { orthophosphate }(\mathrm{pH} 3.5 \text { with OPA): } \\
\text { Acetonitrile }=50: 50, \mathrm{v} / \mathrm{v}\end{array}$ & $\begin{array}{l}\text { Princeton Spher } \mathrm{C}_{18} \\
(250 \times 4.6 \mathrm{~mm}, 5 \mu \mathrm{m})\end{array}$ & 1.0 & 254 & $\begin{array}{l}\text { Pharmaceutical dosage } \\
\text { form }\end{array}$ & [83] \\
\hline & $\begin{array}{l}\text { Bicarbonate buffer }(0.05 \mathrm{M}, \mathrm{pH} 10) \text { : } \\
\text { Acetonitrile }=70: 30, \mathrm{v} / \mathrm{v}\end{array}$ & $\begin{array}{l}\mathrm{X} \text {-Terra } \mathrm{RP}_{18} \\
(4.6 \times 150 \mathrm{~mm})\end{array}$ & 1.0 & 220 & Pharmaceutical product & {$[84]$} \\
\hline & $\begin{array}{l}\text { Mobile phase A: Triethylamine } \\
(0.2 \%, \text { pH } 3 \text { with OPA) buffer } \\
\text { Mobile phase B: Acetonitrile } \\
\text { Gradient programme }\end{array}$ & $\begin{array}{l}\text { Kromasil } C_{18} \\
(250 \times 4.6 \mathrm{~mm}, 5 \mu \mathrm{m})\end{array}$ & 1.0 & 215 & Quality control sample & [85] \\
\hline & $\begin{array}{l}\text { Potassium dihydrogen } \\
\text { orthophosphate }(0.05 \mathrm{mmol}, \mathrm{pH} 3 \\
\text { with OPA): Methanol: Acetonitrile = } \\
60: 25: 15, \mathrm{v} / \mathrm{v} / \mathrm{v}\end{array}$ & $\begin{array}{l}\text { Oyster RP18e } \\
(250 \times 4.6 \mathrm{~mm}, 5 \mu \mathrm{m})\end{array}$ & 1.0 & 207 & Bulk and dosage form & [86] \\
\hline & $\begin{array}{l}\text { Phosphate buffer }(0.05 \mathrm{M}, \mathrm{pH} 3) \\
\text { with triethylamine }(1 \mathrm{ml} / \mathrm{l}): \\
\text { Acetonitrile }=70: 30, \mathrm{v} / \mathrm{v}\end{array}$ & $\begin{array}{l}\text { Shimpack ODS } \\
(150 \times 4.6 \mathrm{~mm}, 5 \mu \mathrm{m})\end{array}$ & 1.6 & 215 & Human serum & {$[87]$} \\
\hline & $\begin{array}{l}\text { Formic acid }(0.04 \mathrm{M}, \mathrm{pH} 3 \text { with } \\
\mathrm{NaOH}): \text { Methanol = 50:50, v/v }\end{array}$ & $\begin{array}{l}\text { Zorbax CN }(150 \times 4.6 \\
\mathrm{mm}, 5 \mu \mathrm{m})\end{array}$ & 1.2 & 226 & Capsule & [88] \\
\hline \multirow[t]{5}{*}{ Zanamivir } & Methanol: Water $=95: 5, \mathrm{v} / \mathrm{v}$ & $\begin{array}{l}\text { Sunfire } C_{18}(4.6 \times 250 \\
\mathrm{mm}, 5 \mu \mathrm{m})\end{array}$ & 1.0 & 285 & Tablet dosage form & [89] \\
\hline & $\begin{array}{l}\text { Phosphate buffer }(0.02 \mathrm{M}, \mathrm{pH} 5) \text { : } \\
\text { Methanol }=50: 50, \mathrm{v} / \mathrm{v}\end{array}$ & $\begin{array}{l}\text { Agilent zorbax } \\
\text { eclipse } C_{18}(150 \times 4.6 \\
\mathrm{mm}, 5 \mu \mathrm{m})\end{array}$ & 1.0 & 230 & Bulk and capsule & [90] \\
\hline & $\begin{array}{l}\text { Potassium dihydrogen } \\
\text { orthophosphate }(\mathrm{pH} 4) \text { : Acetonitrile } \\
=40: 60, \mathrm{v} / \mathrm{v}\end{array}$ & $\begin{array}{l}\text { Xterra Symmetry } C_{8} \\
(4.6 \times 150 \mathrm{~mm}, 3.5 \\
\mu \mathrm{m})\end{array}$ & 0.5 & 230 & Tablets & [91] \\
\hline & $\begin{array}{l}\text { Ultrapure water: Acetonitrile }=98: 2 \text {, } \\
\mathrm{v} / \mathrm{v}\end{array}$ & $\begin{array}{l}\text { BDS Hypersil Cyano } \\
(250 \times 4.6 \mathrm{~mm} ; 5 \\
\mu \mathrm{m})\end{array}$ & 0.5 & 230 & Bulk drug & [92] \\
\hline & Acetonitrile: Water $=50: 50, \mathrm{v} / \mathrm{v}$ & $\begin{array}{l}\text { Supelcocil } \mathrm{C}_{18} \\
(150 \times 4.6 \mathrm{~mm}, 5 \mu \mathrm{m})\end{array}$ & 1.2 & 230 & $\begin{array}{l}\text { Human plasma and } \\
\text { pharmaceutical } \\
\text { formulations }\end{array}$ & [93] \\
\hline \multirow[t]{5}{*}{ Amantadine } & $\begin{array}{l}\text { Mobile phase A: Acetonitrile (5\%) in } \\
\text { water } \\
\text { Mobile phase B: Acetonitrile } \\
\text { Gradient programme }\end{array}$ & $\begin{array}{l}\text { Hypersil } \mathrm{C}_{18} \\
(150 \times 4.6 \mathrm{~mm}, 5 \mu \mathrm{m})\end{array}$ & 1.0 & $\begin{array}{l}\lambda \operatorname{ex} 262 \\
\lambda \operatorname{em} 430\end{array}$ & Rat plasma & [94] \\
\hline & $\begin{array}{l}\text { Ammonium acetate buffer }(0.02 \mathrm{M}) \text { : } \\
\text { Methanol }=12: 88, \mathrm{v} / \mathrm{v}\end{array}$ & $\begin{array}{l}\text { Inertsil ODS-3V } \\
(250 \times 4.6 \mathrm{~mm}, 5 \mu \mathrm{m})\end{array}$ & 1.5 & 226 & Bulk and formulation & [95] \\
\hline & Water: Acetonitrile $=40: 60, \mathrm{v} / \mathrm{v}$ & $\begin{array}{l}\text { Phenomenex RP } \mathrm{C}_{18} \\
(250 \times 4.6 \mathrm{~mm}, 5 \\
\mathrm{mm})\end{array}$ & 1.0 & 273 & Bulk and dosage form & [96] \\
\hline & $\begin{array}{l}\text { Acetonitrile: Sodium acetate buffer } \\
\text { (10 mmol, pH } 3.5 \text { with acetic acid): } \\
\text { Methanol = } 20: 70: 10, \mathrm{v} / \mathrm{v} / \mathrm{v}\end{array}$ & $\begin{array}{l}\text { Nucleosil CN (250x } \\
4.6 \mathrm{~mm}, 5 \mu \mathrm{m})\end{array}$ & 1.5 & $\begin{array}{l}\lambda \operatorname{ex} 293 . \\
\lambda \operatorname{em} 382\end{array}$ & Human plasma & [97] \\
\hline & $\begin{array}{l}\text { Mobile phase A: } 0.1 \% \\
\text { trimethylamine solution (pH 3) }\end{array}$ & $\begin{array}{l}\text { Diamonsil } \mathrm{C}_{18}(200 \mathrm{x} \\
4.6 \mathrm{~mm}, 5 \mu \mathrm{m})\end{array}$ & 1.0 & 210,280 & Quality control granules & [98] \\
\hline
\end{tabular}




\begin{tabular}{|c|c|c|c|c|c|c|}
\hline \multirow{5}{*}{ Rimantadine } & \multicolumn{5}{|l|}{$\begin{array}{l}\text { Mobile phase B: Acetonitrile } \\
\text { Gradient programme }\end{array}$} & [99] \\
\hline & $\begin{array}{l}\text { Methanol: Acetic acid (pH 7) with } \\
\text { water }=4: 1, \mathrm{v} / \mathrm{v}\end{array}$ & $\begin{array}{l}\text { Kanto } C_{18}(150 \times 4.6 \\
\mathrm{mm}, 5 \mu \mathrm{m})\end{array}$ & 0.8 & $\begin{array}{l}\lambda \text { ex } 342 \\
\lambda \text { em } 410\end{array}$ & Rat plasma & [100] \\
\hline & $\begin{array}{l}\text { Ethylnitrile and octane sulfonic acid } \\
\text { buffer }(0.005 \mathrm{M}, \mathrm{pH} 6.7)=60: 40, \mathrm{v} / \mathrm{v}\end{array}$ & $\mathrm{C}_{18}(250 \times 4.6 \mathrm{~mm})$ & 1.0 & 259 & Medicinal form & [101] \\
\hline & $\begin{array}{l}\text { СH3OH: phosphate buffer, } 25 \\
\mathrm{mmol} / \mathrm{l}(\mathrm{pH} 3)=50: 50, \mathrm{v} / \mathrm{v}\end{array}$ & $\begin{array}{l}\text { Monolithic RP } \\
(100 \times 4.6 \mathrm{~mm})\end{array}$ & 0.5 & $\begin{array}{l}\lambda \text { ex } 340 \\
\lambda \text { em } 455\end{array}$ & Human urine & [102] \\
\hline & $\begin{array}{l}\text { Methanol: Acetic acid (pH 7) with } \\
\text { water }=4: 1, \mathrm{v} / \mathrm{v}\end{array}$ & $\begin{array}{l}\text { Kanto } \mathrm{C}_{18}(150 \times 4.6 \\
\mathrm{mm}, 5 \mu \mathrm{m})\end{array}$ & 0.8 & $\begin{array}{l}\lambda \text { ex } 342 \\
\lambda \text { em } 410 \\
\end{array}$ & Rat plasma & [100] \\
\hline
\end{tabular}

\section{Ultra pressure liquid chromatography}

Ultra pressure liquid chromatography (UPLC) is obligatory in respect to the analysis time; resolution enhanced its reliability in the pharmaceutical industry. The achieved data from developed methods are robust and accurate. The common available columns for UPLC are acquity $\mathrm{BEH} \mathrm{C}_{8}$, acquity $\mathrm{BEH} \mathrm{C}_{18}$, acquity $\mathrm{BEH} \mathrm{RP}_{18}$ and acquity BEH phenyl. UV-Visible detector is commonly used detector for UPLC analysis. The method for Oseltamivir and its degraded product is established within 5 minute using mobile phase composition phosphate buffer, $\mathrm{pH} 3.5$ and methanol $(80: 20, \mathrm{v} / \mathrm{v}$ ), detected at $207 \mathrm{~nm}$ [108].

\section{Liquid chromatography-mass spectrophotometry}

Liquid chromatography-mass spectrophotometry technique is involved in the physical separation of compounds by liquid chromatography (LC) combined mass spectrometry (MS) for mass analysis [109-113]. Liquid chromatography can be high pressure or ultra-pressure. The structure of molecule elucidate after confirming the chemical composition of charged particles with respect to massto-charge ratio. The molecule ionized by MS to produce fragments and its mass-to-charge ratio measured. This technique maintains high sensitivity compared to traditional techniques and selective to many applications. It has an excellent impact in the bioanalysis field mainly confronted to pharmacokinetics investigation of pharmaceuticals. Generally, LC installed octadecyl $\left(\mathrm{C}_{18}\right)$ silica column but nowadays hydrophilic interaction based liquid chromatography (HILIC) has received more attention and recommended due to its potential to retain highly polar molecule with hydrophilic nature, but it needs to apply buffer as the mobile phase to minimize analyte and stationary phase ionic interaction. The LCMS method that was reported for inhibitors is presented in table 2 [114-137].

Table 2: LCMS method for neuraminidase and M2 ion channel inhibitors

\begin{tabular}{|c|c|c|c|c|c|c|}
\hline Mobile phase & Stationary phase & Sample & $\begin{array}{l}\text { Flow rate } \\
(\mathrm{ml} / \mathrm{min})\end{array}$ & $\begin{array}{l}\text { Run } \\
\text { time(min) }\end{array}$ & Application & Reference \\
\hline $\begin{array}{l}\text { Ammonium formate }(10 \mathrm{mmol} \text {, } \\
\mathrm{pH} 3) \text { : Acetonitrile }=20: 80, \mathrm{v} / \mathrm{v}\end{array}$ & $\begin{array}{l}\text { Synergi } C_{18}(150 \times 4.6 \\
\mathrm{mm}, 4 \mu \mathrm{m})\end{array}$ & Amantadine & 0.8 & 2.5 & Human plasma & {$[114]$} \\
\hline $\begin{array}{l}\text { Mobile phase A: Ammonium } \\
\text { formate ( } 5 \mathrm{mmol} \text {, in water) } \\
\text { Mobile phase B: Acetonitrile; } \\
\text { Gradient programme }\end{array}$ & $\begin{array}{l}\text { Eclipse Plus } C_{18} \\
(50 \times 3 \mathrm{~mm}, 3.5 \mu \mathrm{m})\end{array}$ & Amantadine & --- & 5.3 & Human plasma & [115] \\
\hline $\begin{array}{l}\text { Mobile phase A: Ammonium } \\
\text { formate }(10 \mathrm{mmol} / \mathrm{l}, 0.1 \% \\
\text { formic acid) } \\
\text { Mobile phase B: Acetonitrile; } \\
\text { Gradient programme }\end{array}$ & $\begin{array}{l}\text { Kinetex XB } \mathrm{C}_{18}(2.1 \times \\
100 \mathrm{~mm}, 2.6 \mu \mathrm{m})\end{array}$ & $\begin{array}{l}\text { Amantadine, } \\
\text { Rimantadine }\end{array}$ & 0.3 & 15 & $\begin{array}{l}\text { Chicken tissue and } \\
\text { eggs }\end{array}$ & {$[116]$} \\
\hline $\begin{array}{l}\text { Mobile phase A: Formic acid } \\
(0.1 \%) \text { in water } \\
\text { Mobile phase B: Acetonitrile; } \\
\text { Gradient programme }\end{array}$ & $\begin{array}{l}\text { Eclipse Plus } C_{18} \\
(100 \times 2.1 \mathrm{~mm}, 1.8 \\
\mu \mathrm{m}) \text { Zorbax SB C } 18 \\
(100 \times 2.1 \mathrm{~mm}, 3.5 \\
\mu \mathrm{m})\end{array}$ & $\begin{array}{l}\text { Amantadine, } \\
\text { Rimantadine }\end{array}$ & 0.35 & 5 & Chicken jerky & [117] \\
\hline $\begin{array}{l}\text { Mobile phase A: Formic acid } \\
(0.1 \%) \text { in water } \\
\text { Mobile phase B: Formic acid } \\
(0.1 \%) \text { in acetonitrile; Gradient } \\
\text { programme }\end{array}$ & $\begin{array}{l}\text { ZIC-HILIC }(50 \times 2.1 \\
\mathrm{mm}, 5 \mu \mathrm{m})\end{array}$ & Zanamivir & 0.4 & 15 & $\begin{array}{l}\text { Milli-Q water, } \\
\text { sewage effluent, } \\
\text { influent and surface } \\
\text { water }\end{array}$ & [118] \\
\hline $\begin{array}{l}\text { Acetonitrile: } 0.1 \% \text { Formic acid = } \\
90: 10, \mathrm{v} / \mathrm{v}\end{array}$ & $\begin{array}{l}\text { Hydrosphere } C_{18} \\
(150 \times 4.6 \mathrm{~mm}, 5 \mu \mathrm{m})\end{array}$ & $\begin{array}{l}\text { Oseltamivir and } \\
\text { oseltamivir } \\
\text { carboxylic acid }\end{array}$ & 0.5 & 5.5 & Human plasma & [119] \\
\hline $\begin{array}{l}\text { Mobile phase A: Formic acid } \\
(0.1 \%) \text { in water } \\
\text { Mobile phase B: Methanol; } \\
\text { Gradient programme }\end{array}$ & $\begin{array}{l}\mathrm{XDB} \mathrm{C}_{18}(2.1 \times 150 \\
\mathrm{mm}, 3.5 \mu \mathrm{m})\end{array}$ & $\begin{array}{l}\text { Amantadine, } \\
\text { Rimantadine }\end{array}$ & 0.3 & 10 & $\begin{array}{l}\text { Chicken, pig, pork, } \\
\text { duck (liver, kidney, } \\
\text { egg) }\end{array}$ & {$[120]$} \\
\hline $\begin{array}{l}\text { Mobile phase A-Methanol: } \\
\text { Water=1:99, v/v } \\
\text { Mobile phase B-Methanol: } \\
\text { Water=99:1, v/v } \\
\text { Gradient programme }\end{array}$ & $\begin{array}{l}\text { Eclipse Plus } C_{18} \\
(50 \times 2.1 \mathrm{~mm}, 5 \mu \mathrm{m})\end{array}$ & $\begin{array}{l}\text { Oseltamivir and } \\
\text { oseltamivir } \\
\text { carboxylate }\end{array}$ & --- & 4.5 & Human plasma & {$[121]$} \\
\hline $\begin{array}{l}\text { Ammonium formate }(10 \mathrm{mmol}) \text { : } \\
\text { Acetonitrile }=30: 70, \mathrm{v} / \mathrm{v}\end{array}$ & $\begin{array}{l}\text { Symmetry } C_{18} \\
(100 \times 4.6 \mathrm{~mm}, 5 \mu \mathrm{m})\end{array}$ & $\begin{array}{l}\text { Oseltamivir and } \\
\text { oseltamivir } \\
\text { carboxylate }\end{array}$ & 1.0 & 2 & Human plasma & [122] \\
\hline Ammonium acetate $(10 \mathrm{mmol}$, & ZIC-HILIC $(150 \times 2.1$ & Oseltamivir and & 0.3 & 10 & Rat Plasma & [123] \\
\hline
\end{tabular}




\begin{tabular}{|c|c|c|c|c|c|c|}
\hline $\mathrm{pH}$ 6): Acetonitrile $=17: 83, \mathrm{v} / \mathrm{v}$ & $\mathrm{mm}, 3.5 \mu \mathrm{m})$ & $\begin{array}{l}\text { oseltamivir } \\
\text { carboxylic acid }\end{array}$ & & & & \\
\hline $\begin{array}{l}\text { Mobile phase A: Formic acid ( } 50 \\
\text { mmol) in water }\end{array}$ & $\begin{array}{l}\text { Symmetry } C_{18} \\
(3 \times 150 \mathrm{~mm}, 5 \mu \mathrm{m})\end{array}$ & $\begin{array}{l}\text { Oseltamivir, } \\
\text { oseltamivir }\end{array}$ & 0.4 & 20 & Poultry muscle & [124] \\
\hline $\begin{array}{l}\text { Mobile phase B: Formic acid ( } 50 \\
\text { mmol) in acetonitrile; Gradient } \\
\text { programme }\end{array}$ & $\begin{array}{l}\text { Hypercarb }(3 \times 100 \\
\mathrm{mm}, 5 \mu \mathrm{m})\end{array}$ & $\begin{array}{l}\text { carboxylate, } \\
\text { zanamivir, } \\
\text { amantadine, } \\
\text { rimantadine }\end{array}$ & & & & \\
\hline $\begin{array}{l}\text { Mobile phase A: Formic acid } \\
(0.1 \%) \text { Mobile phase B: } \\
\text { Methanol; Gradient programme }\end{array}$ & $\begin{array}{l}\text { Synergi } \mathrm{C}_{18}(150 \times 2.0 \\
\mathrm{mm}, 4 \mu \mathrm{m})\end{array}$ & $\begin{array}{l}\text { Oseltamivir and } \\
\text { oseltamivir } \\
\text { carboxylate }\end{array}$ & 0.25 & 9 & Human plasma & [125] \\
\hline $\begin{array}{l}\text { Mobile phase A: Ammonium } \\
\text { acetate (10 mmol, } 1 \% \text { acetic } \\
\text { acid) Mobile phase B: } \\
\text { Acetonitrile; Gradient } \\
\text { programme }\end{array}$ & $\begin{array}{l}\text { ZIC-HILIC (50x2.1 } \\
\mathrm{mm}, 5 \mu \mathrm{m})\end{array}$ & Zanamivir & 0.5 & 4 & Human plasma & [126] \\
\hline $\begin{array}{l}\text { Ammonium acetate }(10 \mathrm{mmol} \text {, } \\
\mathrm{pH} 3.5) \text { : Formic acid }(0.1 \%) \text { in } \\
\text { acetonitrile }=20: 80, \mathrm{v} / \mathrm{v}\end{array}$ & $\begin{array}{l}\text { Chromatopack } \mathrm{C}_{18} \\
(50 \times 3 \mathrm{~mm}, 3.0 \mu \mathrm{m})\end{array}$ & $\begin{array}{l}\text { Oseltamivir and } \\
\text { oseltamivir } \\
\text { carboxylate }\end{array}$ & 0.6 & 1 & Human plasma & [127] \\
\hline $\begin{array}{l}\text { Mobile phase A: Ammonium } \\
\text { acetate ( } 10 \mathrm{mmol}, 1 \% \\
\text { methanol) Mobile phase B: } \\
\text { Acetonitrile; Gradient } \\
\text { programme }\end{array}$ & $\begin{array}{l}\text { ZIC-HILIC (50x2.1 } \\
\mathrm{mm}, 3 \mu \mathrm{m})\end{array}$ & Zanamivir & 0.3 & 5 & $\begin{array}{l}\text { Rat and monkey } \\
\text { plasma }\end{array}$ & [128] \\
\hline $\begin{array}{l}\text { Water: Acetonitrile }=60: 40 \mathrm{v} / \mathrm{v} \text {, } \\
\text { with } 5 \mathrm{~g} / \mathrm{l} \text { formic acid }\end{array}$ & $\begin{array}{l}\text { Phenomenex Luna } \\
\mathrm{C}_{8}(100 \times 2 \mathrm{~mm}, 3 \\
\mu \mathrm{m})\end{array}$ & Amantadine & 0.2 & 3 & Human serum & [129] \\
\hline $\begin{array}{l}\text { Mobile phase A: Formic acid } \\
(0.1 \%) \text { in water } \\
\text { Mobile phase B: Methanol; } \\
\text { Gradient programme }\end{array}$ & $\begin{array}{l}\text { XDB } C_{18}(2.1 \times 150 \\
\mathrm{mm}, 3.5 \mu \mathrm{m})\end{array}$ & $\begin{array}{l}\text { Amantadine, } \\
\text { Rimantadine }\end{array}$ & 0.3 & 10 & $\begin{array}{l}\text { Feed pigs and } \\
\text { chicken }\end{array}$ & {$[130]$} \\
\hline $\begin{array}{l}\text { Mobile phase A: Formic acid } \\
(0.1 \% \text { solution) } \\
\text { Mobile phase B: Methanol; } \\
\text { Gradient programme }\end{array}$ & $\begin{array}{l}\text { Agilent SB-Aq (3× } \\
100 \mathrm{~mm}, 1.8 \mu \mathrm{m})\end{array}$ & $\begin{array}{l}\text { Amantadine, } \\
\text { oseltamivir, } \\
\text { rimantadine, } \\
\text { oseltamivir } \\
\text { carboxylate }\end{array}$ & 0.3 & 11 & $\begin{array}{l}\text { Chicken muscle } \\
\text { tissues }\end{array}$ & [131] \\
\hline $\begin{array}{l}\text { Mobile phase A: Formic acid } \\
(0.1 \%) \text { in water } \\
\text { Mobile phase B: Acetonitrile; } \\
\text { Gradient programme }\end{array}$ & $\begin{array}{l}\mathrm{BEH} \mathrm{C}_{18}(2.1 \times 100 \\
\mathrm{mm}, 1.7 \mu \mathrm{m})\end{array}$ & $\begin{array}{l}\text { Peramivir, } \\
\text { laninamivir, } \\
\text { oseltamivir, } \\
\text { oseltamivir } \\
\text { carboxylate, } \\
\text { zanamivir }\end{array}$ & 0.35 & 8 & $\begin{array}{l}\text { River waters, } \\
\text { sewage effluent }\end{array}$ & [132] \\
\hline $\begin{array}{l}\text { Mobile phase A: Formic acid } \\
(0.1 \% \text { solution) } \\
\text { Mobile phase B: Acetonitrile; } \\
\text { Gradient programme }\end{array}$ & $\begin{array}{l}\text { Acquity HSS T3 } \\
(150 \times 2.1 \mathrm{~mm}, 1.8 \\
\mu \mathrm{m})\end{array}$ & $\begin{array}{l}\text { Zanamivir, } \\
\text { Oseltamivir }\end{array}$ & 0.7 & 8.5 & $\begin{array}{l}\text { Waste and surface } \\
\text { water }\end{array}$ & [133] \\
\hline $\begin{array}{l}\text { Mobile phase A: Ammonium } \\
\text { acetate ( } 10 \mathrm{mmol}), 5 \% \\
\text { acetonitrile adjusted pH } 5 \text { with } \\
\text { acetic acid Mobile phase B: } \\
\text { Ammonium acetate }(10 \mathrm{mmol}) \text {, } \\
\text { pH } 5 \text { with acetic acid; Gradient } \\
\text { programme }\end{array}$ & $\begin{array}{l}\text { Acquity HILIC } \\
(100 \times 2.1 \mathrm{~mm}, 1.7 \\
\mu \mathrm{m})\end{array}$ & $\begin{array}{l}\text { Osetamivir } \\
\text { phosphate, } \\
\text { oseltamivir } \\
\text { carboxylate }\end{array}$ & 0.7 & 8.5 & River water & [134] \\
\hline $\begin{array}{l}\text { Mobile phase A: Formic acid } \\
(0.1 \% \text { solution) } \\
\text { Mobile phase B: Acetonitrile; } \\
\text { Gradient programme }\end{array}$ & $\begin{array}{l}\text { Acquity HSS T3 } \\
(150 \times 2.1 \mathrm{~mm}, 1.8 \\
\mu \mathrm{m})\end{array}$ & $\begin{array}{l}\text { Amantadine, } \\
\text { Rimantadine }\end{array}$ & 0.25 & 12 & Chicken muscle & [135] \\
\hline $\begin{array}{l}\text { Mobile phase A: Formic acid } \\
(0.1 \%) \text { in water } \\
\text { Mobile phase B: Methanol; } \\
\text { Gradient programme }\end{array}$ & $\begin{array}{l}\mathrm{BEH} \mathrm{C}_{18}(2.1 \times 100 \\
\mathrm{mm}, 1.7 \mu \mathrm{m})\end{array}$ & $\begin{array}{l}\text { Amantadine, } \\
\text { Rimantadine }\end{array}$ & 0.3 & 10 & Chicken muscle & [136] \\
\hline $\begin{array}{l}\text { Mobile phase A: Formic acid } \\
(0.1 \%) \text { in water } \\
\text { Mobile phase B: Formic acid } \\
(0.1 \%) \text { in methanol; Gradient } \\
\text { programme }\end{array}$ & $\begin{array}{l}\text { Ascentis C18 } \\
(100 \times 2.1 \mathrm{~mm} \text {, } \\
2.7 \mu \mathrm{m})\end{array}$ & $\begin{array}{l}\text { Oseltamivir, } \\
\text { oseltamivir } \\
\text { carboxylate }\end{array}$ & 0.35 & 6 & Human plasma & [137] \\
\hline
\end{tabular}

The LCMS/MS investigation revealed that low concentration of amantadine present in plasma sample, does not require any derivatization during solid phase extraction, the developed method reported successfully bioequivalence study of healthy volunteers [114]. Amantadine-d15 internal standard can be used based on protein precipitation [115]. QuEChERS extraction method applied for chicken muscle, egg, pet treat sample [116, 117]. Zanamivir found in effluent sample and water from river in Japan [118]. The antiviral drugs and metabolite quantified applied flow rate $(0.2-1$ $\mathrm{ml} / \mathrm{min}$ ) having run time between 1-20 minute with biological fluids and water sample [119-129]. Ultra pressure liquid chromatography combined with the mass spectrophotometer (triple quadrupole ion 
trap) reported in feed sample illegal addition of amantadine and rimantadine [130]. Fourteen antiviral drugs detected within short time of 11 minute, multiple reaction monitoring ensured method specificity [131]. After winter season the inhibitors found in river water [132-134]. Amantadine and rimantadine are quantified in chicken muscle using different sorbent [135-136]. The UPLC method was first reported with whole blood sample, assay calculated using dried blood spot [137]. The minimum amount of sample required, elute from the column within 6 minute. The correlation coefficient of Oseltamivir and its active metabolites Oseltamivir carboxylate was greater than 0.99. Sample collection is very important parameter for this analysis. Therefore, the developed method validated successfully for human plasma of healthy volunteers.

\section{CONCLUSION}

Antiviral drugs are very useful versus influenza viruses and molecules that are actively participated for deadly diseases. The developed novel drugs are showing great potential for the prevention of influenza. It was evident that for controlling influenza, administered single dose drug to human and all animals including farm animals is required. All inhibitors can be applied during epidemic or seasonal conditions. Their main function, through vaccination, is to control the activity of viruses, reduce and manage pandemic viruses. All these mentioned drugs are also important for patients hospitalized due to influenza. Although the existing drugs are doing well, influenza experts are looking forward to identify other new drugs that exert antiviral activity like the approved ones and can be applicable as future antiviral agents for influenza viruses. Therefore, the present review gives brief information about antiviral drugs, their activity, biotransformation and analytical methods for quantification and this information will be helpful for any future studies done by experts in this field.

\section{ACKNOWLEDGMENT}

The authors of this review would like to thank Jubail Industrial College management team and JIC-RPPAP team for their continuous help and support.

\section{AUTHORS CONTRIBUTIONS}

All the author have contributed equally

\section{CONFLICT OF INTERESTS}

Both authors report no declaration of interest

\section{REFERENCES}

1. Zhu HY, Han L, Shi XL, Wang BL, Huang $H$, Wang $X$, et al. Baicalin inhibits autophagy induced by influenza a virus H3N2. Antiviral Res 2015;113:62-70.

2. Fiore AE, Shay DK, Broder K, Iskander JK, Uyeki TM, Mootrey G, et al. Centers for disease control and prevention (CDC), advisory committee on immunization practices (ACIP). Prevention and control of influenza: recommendations of the advisory committee on immunization practices (ACIP). MMWR Recomm Rep 2008;57(RR-7):1-60.

3. Chang SC, Cheng YY, Shih SR. Avian influenza virus: the threat of a pandemic. Chang Gung Med J 2006;29:130-4.

4. Liu Y, Zhang J, Xu W. Recent progress in rational drug design of neuraminidase inhibitors. Curr Med Chem 2007;14:2872-91.

5. Zhang Z. Spatio-temporal data comparisons for global highly pathogenic avian influenza (HPAI) H5N1 outbreaks. PLoS One 2010;5:e15314.

6. Tanaka T. Safety of neuraminidase inhibitors against novel influenza A (H1N1) in pregnant and breastfeeding women. Can Med Assoc J 2009;181:55-8.

7. Laver WG, Bischofberger N, Webster RG. Disarming flu viruses. Sci Am 1999;280:78-87.

8. Laver WG, Bischofberger N, Webster RG. The origin and control of pandemic influenza. Perspect Biol Med 2000;43:173-92.

9. Palese P, Schulman JL, Bodo G, Meindl P. Inhibition of influenza and parainfluenza virus replication in tissue culture by 2deoxy-2,3-dehydro-N-trifluoro-acetylneuraminic acid (FANA). Virology 1974;59:490-8.
10. Russell RJ. The structure of H5N1 avian influenza neuraminidase suggests new opportunities for drug design. Nature 2006;443:45-9.

11. Pinto LH, Holsinger LJ, Lamb RA. Influenza virus M2 protein has ion channel activity. Cell 1992;69:517-28.

12. Hayden FG. Amantadine and rimantadine-clinical aspects. In: Richman DD. editor. Antiviral Drug Resistance. New York: John Wiley and Sons, Inc; 1996. p. 59-77.

13. Hay AJ, Wolstenholme AJ, Skehel JJ. The molecular basis of the specific anti-influenza action of amantadine. The EMBO J 1985;4:3021-4.

14. Sidwell RW, Huffman JH, Barnard DL. Inhibition of influenza virus infections in mice by GS4104, an orally effective influenza virus neuraminidase inhibitor. Antiviral Res 1998;37:107-20.

15. Monto AS, Fleming DM, Henry D. Efficacy and safety of the neuraminidase inhibitor zanamivirin the treatment of influenza A and B virus infections. J Infect Dis 1999;180:254-61.

16. Monto AS. The role of antivirals in the control of influenza. Vaccine 2003;21:1796-800.

17. McKimm BJL. Management of influenza virus infections with neuraminidase inhibitors: detection, incidence, and implications of drug resistance. Treat Respir Med 2005;4:107-16.

18. Wade RC. Flu and structure-based drug design. Structure 1997;5:1139-45.

19. Gubareva LV, Kaiser L, Hayden FG. Influenza virus neuraminidase inhibitors. Lancet 2000;355:827-35.

20. Laver WG. From the great barrier reef to a "Cure" for the flu: tall tales, but true. Perspect Biol Med 2004;47:590-6.

21. Muthuri SG. Effectiveness of neuraminidase inhibitors in reducing mortality in patients admitted to hospital with influenza A H1N1pdm09 virus infection: a meta-analysis of individual participant data. Lancet Resp Med 2014;2:395-404.

22. Heneghan CJ. Neuraminidase inhibitors for influenza: a systematic review and meta-analysis of regulatory and mortality data. Health Technol Assess 2016;20:1-242.

23. Luo M. Structural biology: antiviral drugs fit for a purpose. Nature 2006;443:37-38.

24. McLaughlin MM, Skoglund EW, Ison MG. Peramivir: an intravenous neuraminidase inhibitor. Expert Opin Pharmacother 2015;16:1889-900.

25. Yamashita M. CS-8958, a prodrug of the new neuraminidase inhibitor R-125489, shows long-acting anti-influenza virus activity. Antimicrob Agents Chemother 2009;53:186-92.

26. Burger RA. Immunological effects of the orally administered neuraminidase inhibitor oseltamivir in influenza virus-infected and uninfected mice. Immun Pharma 2000;47:45-52.

27. Klumpp K, Graves BJ. Optimization of small molecule drugs binding to highly polar target sites: lessons from the discovery and development of neuraminidase inhibitors. Curr Top Med Chem 2006;6:423-34.

28. Vavricka CJ. Structural and functional analysis of laninamivir and its octanoate prodrug reveals group specific mechanisms for influenza NA inhibition. PLoS Pathog 2011;7:e1002249.

29. Bright RA, Medina MJ, Xu X, Perez Oronoz G, Wallis TR. Incidence of adamantane resistance among influenza A (H3N2) viruses isolated worldwide from 1994 to 2005: a cause for concern. Lancet 2005;366:1175-81.

30. Bright RA, Shay DK, Shu B, Cox NJ, Klimov A. Adamantane resistance among influenza viruses isolated early during the 2005-2006 influenza season in the United States. JAMA: J Am Med Assoc 2006;295:891-4.

31. Duque MD, Valverde E, Barniol M, Guardiola S, Rey M. Inhibitors of the M2 channel of influenza a virus. Recent Adv Pharm Sci 2011;2:35-64.

32. Govorkova EA, McCullers JA. Therapeutics against influenza. Curr Top Microbiol Immunol 2013;370:273-300.

33. Helenius A. Unpacking the incoming influenza virus. Cell 1992;69:577-8.

34. Wattanagoon Y, Stepniewska K, Lindegardh N. Pharmacokinetics of high-dose oseltamivir in healthy volunteers. Antimicrob Agents Chemother 2009;53:945-52.

35. Dutkowski R, Smith JR, Davies BE. Safety and pharmacokinetics of oseltamivir at standard and high dosages. Int J Antimicrob Agents 2010;35:461-7. 
36. Sharma PP, Roy RK, Anurag. Neuraminidase inhibitors: Oseltamivir, Peramivir, synthesis and profile. J Pharm Res 2010;3:1602-6.

37. He G, Massarella J, Ward P. Clinical pharmacokinetics of the prodrug oseltamivir and its active metabolite Ro 64-0802. Clin Pharmacokinet 1999;37:471-84.

38. Doucette KE, Aoki FY. Oseltamivir: a clinical and pharmacological perspective. Expert Opin Pharmacother 2001;2:1671-83.

39. Glaxo Smith Kline. Safety study to assess IV zanamivir for treatment of influenza infection in patients who are in hospital (NAI113678). Available from: http://clinicaltrials. gov/ct2/show/NCT01014988. [Last accessed on 11 Feb 2011]

40. Glaxo Smith Kline. A study of intravenous zanamivir versus oral oseltamivir in adults and adolescents hospitalized with influenza (ZORO). Available from: http://clinicaltrials.gov/ ct2/show/NCT01231620. [Last accessed on 11 Feb 2011]

41. Cass LM, Brown J, Pickford M. Pharmacoscintigraphic evaluation of lung deposition of inhaled zanamivir in healthy volunteers. Clin Pharmacokinet 1999;36(Suppl 1):21-31.

42. Bergstrom M, Cass LM, Valind S. Deposition and disposition of [11C] zanamivir following administration as an intranasal spray: evaluation with positron emission tomography. Clin Pharmacokinet 1999;36(Suppl 1):33-9.

43. Peng AW, Milleri S, Stein DS. Direct measurement of the antiinfluenza agent zanamivir in the respiratory tract following inhalation. Antimicrob Agents Chemother 2000;44:1974-6.

44. Calfee DP, Peng AW, Cass LM, Lobo M, Hayden FG. Safety and efficacy of intravenous zanamivir in preventing experimental human influenza a virus infection. Antimicrob Agents Chemother 1999;43:1616-20.

45. Bantia S, Parker CD, Ananth SL, et al. Comparison of the antiinfluenza virus activity of RWJ-270201 with those of oseltamivir and zanamivir. Antimicrob Agents Chemother 2001;45:1162-7.

46. Kohno S, Kida H, Mizuguchi M, Shimada J. Efficacy and safety of intravenous peramivir for treatment of seasonal influenza virus infection. Antimicrob Agents Chemother 2010;54:4568-74.

47. Itoh $Y$, Shinya K, Kiso M. In vitro and in vivo characterization of new swine-origin H1N1 influenza viruses. Nature 2009;460:1021-5.

48. Yamashita M, Tomozawa T, Kakuta M, Tokumitsu A, Nasu H, Kubo S. CS-8958, a prodrug of the new neuraminidase inhibitor R-125489, shows long-acting anti-influenza virus activity. Antimicrob Agents Chemother 2009;53:186-92.

49. Yamashita M. Laninamivir and its prodrug, CS-8958:long-acting neuraminidase inhibitors for the treatment of influenza. Antivir Chem Chemother 2010;21:71-84.

50. Koyama K, Ogura Y, Nakai D, Watanabe M, Munemasa T, Oofune $\mathrm{Y}$, et al. Identification of bioactivating enzymes involved in the hydrolysis of laninamivir octanoate, a long-acting neuraminidase inhibitor, in human pulmonary tissue. Drug Metab Dispos 2014;42:1031-8.

51. B1eidner WE, Harmon JB, Hewes WE, Lynes TE, Hermann EC. Absorption, distribution and excretion of amantadine hydrochloride. J Pharmacol Exp Ther 1965;150:484-90.

52. Montanari C, Ferrari P, Bavazzano A. Urinary excretion of amantadine by the elderly. Eur J Clin Pharm 1975;8:349-51.

53. Koppel C, Tenczer J. A revision of the metabolic disposition of Amantadine. Biomed Mass Spectro 1985;12:499-501.

54. Hayden FG, Minocha A, Spyker DA, Hoffman HE. Comparative single dose pharmacokinetics of amantadine hydrochloride and rimantadine hydrochloride in young and elderly adults. Antimicrob Agents Chemother 1985;28:216-21.

55. Wills RJ. The clinical pharmacokinetics of rimantadine. J Respir Dis 1987;8(Suppl 11A):S39-44.

56. Capparelli EV, Stevens RC, Chow MS, Izard M, Wills RJ. Rimantadine pharmacokinetics in healthy subjects and patients with end-stage renal failure. Clin Pharmacol Ther 1988;43:536-41.

57. Rubio FR, Fukuda EK, Garland WA. Urinary metabolites of rimantadine in humans. Drug Metab Dispos Biol Fate Chern 1988;16:773-7.

58. Wills RJ, Belshe R, Tomlinsin D, De Grazia F, Lin A, Wells S. Pharmacokinetics of rimantadine hydrochloride in patients with chronic liver disease. Clin Pharmacol Ther 1987;42:44954.

59. Rubio FR, Fukuda EK, Garland WA. Urinary metabolites of rimantadine in humans. Drug Metab Dispos 1988;16:773-7.

60. Wills RJ. Update on rimantadine's clinical pharmacokinetics. J Respir Dis 1989;10(Suppl):S20-5.

61. Rubio FA, Choma N, Fukuda EK. Determination of rimantadine and its hydroxylated metabolites in human plasma and urine. J Chromatogr 1989;497:147-57.

62. Brown SY, Garland WA, Fukuda EK. Isolation and characterization of an unusual glucuronide conjugate of rimantadine. Drug Metab Dispos Biol Fate Chern 1990;18:546-7.

63. Youssef RM, El-Yazbi EA, Khamis EF, Younis SE. Validated spectrophotometric methods for the evaluation of oseltamivir counterfeit pharmaceutical capsules; Bull Facul Pharm Cairo Uni 2014;52:63-9.

64. Bano T, Yadav G, Dudhe R. Development and validation of oseltamivir phosphate API by UV-spectrophotometer. Global J Pharm 2013;7:294-7.

65. Raut CS, Gharge DS, Dhabale PN, Gonjari ID, Hosmani AH, Hosmani AH. Development and validation of oseltamivir phosphate in fluvir ${ }^{\circledR}$ by UV-spectrophotometer. Int J PharmTech Res 2010;2:363-6.

66. Kumar JVS, Prasanthi S, Guravaiah M, Sekaran CB. Application of potassium permanganate to the spectrophotometric determination of oseltamivir phosphate in bulk and capsules. Asian J Pharm Clin Res 2012;5:18-22.

67. Nebsen M, Fattah SAA, Hassan DW, Youssef NF. Spectrophotometric and spectrofluorimetric determination of oseltamivir phosphate using 4-chloro-7-nitrobenzo-2-oxa 1, 3diazole. Anal Chem Indian J 2011;10:336-41.

68. Reddy CMB, GV Reddy GVS. UV visible spectrophotometric estimation of Zanamvir. J Chem Pharm Res 2017;9:189-92.

69. Reddy CMB, Reddy GVS. A validated UV spectrophotometric determination of an antiviral drug zanamvir from tablet formulations. J Chem Pharm Res 2012;4:3624-7.

70. Omara HA, Amin AS. Extractive-spectrophotometric methods for determination of anti-parkinsonian drug in pharmaceutical formulations and in biological samples using sulphonphthalein acid dyes. J Saudi Chem Soc 2012;16:75-81.

71. Mahmoud AM, Khalil NY, Darwish IA, Aboul-Fadl T. Selective spectrophotometric and spectrofluorimetric methods for the determination of amantadine hydrochloride in capsules and plasma via derivatization with 1, 2-naphthoquinone-4sulphonate. Int J Anal Chem 2009;810104:8. Doi:10.1155/ 2009/810104.

72. Gursoy A, Ozkirimli S, Ersoy 0. Spectrophotometric determination of amantadine hydrochloride in capsules. J Fac Pharm Istanbul 1998;32:63-8.

73. Choi K, Choi JK, Yoo GS. Spectrophotometric determination of amantadine sulfate after ion-pairing with methyl orange. Arch Pharm Res 1991;14:285-9.

74. Muszalska I, Sobczak A, Kiaszewicz I, Rabiega K, Lesniewska MA, Jelińska A. 1, 2-Naphthoquinone-4-sulfonic acid sodium salt as a reagent for spectrophotometric determination of rimantadine and memantine. J Anal Chem 2015;70:320-7.

75. Bano T, Dudhe R, Kumar N. Development and validation of rphplc method for the determination of oseltamivir phosphate API. J Progr Res Chem 2015;2:69-73.

76. Kumar NM, Abbulu K, Narayana BV. Development and validation of stability indicating rp-hplc method for the determination of oseltamivir phosphate in oseltamivir phosphate capsules. Int J Res Chem Environ 2015;5:44-53.

77. Junaidy MAQ, Haque MA, Bakshi V. Rp-hplc method development and validation for the estimation of oseltamivir phosphate in bulk form and pharmaceutical formulations. Int J Innov Pharma Sci Res 2014;2:2786-91.

78. Ameti A, Slavkovska J, Starkoska K, Sarafinovska ZA. A simple isocratic rp-hplc method for quality control of oseltamivir capsules. Maced J Chem Eng 2012;31:205-15.

79. Sharma YK, Agarwal DD, Bhure S, Rathore SS, Rawat C, Mukharjee R. Synthesis, isolation and characterization of process-related impurities in oseltamivir phosphate. E-J Chem 2012;9:113-20. 
80. Malipatil SM, Jahan K, Patil SK. Development and validation of rphplc method for the determination of oseltamivir phosphate in bulk drug and in dosage. Indo-Global J Pharma Sci 2011;1:57-62.

81. Sharma MC, Sharma S. Dissolution assessment and hplc method development and validation of oseltamivir phosphate in pharmaceutical formulation. Afr J Basic Appl Sci 2011;3:323-8.

82. Zeynep A, Sena C, Sidıka T. Rp-hplc method for determination of oseltamivir phosphate in capsules and spiked plasma. Anal Lett 2010;43:2200-9.

83. Nagarajan JSK, Muralidharan S. A validated RP-HPLC method for estimation of Oseltamivir in pharmaceutical formulation. Der Pharm Lett 2009;1:162-8.

84. Green MD, Nettey H, Wirtz RA. Determination of oseltamivir quality by colorimetric and liquid chromatographic methods. Emer Infect Dis 2008;14;552-6.

85. Narasimhan B, Abida K, Srinivas K. Stability indicating RP-HPLC method development and validation for oseltamivir API. Chem Pharm Bull 2008;56:413-7.

86. Raghuram P, Raju IVS, Reddy R, Sriramulu J. A stability indicating LC method for oseltamivir phosphate. Anal Chem Indian J 2008;7:617-24.

87. Bahrami G, Mohammadi B, Kiani A. Determination of oseltamivir carboxylic acid in human serum by solid phase extraction and high performance liquid chromatography with UV detection. J Chromatogr B 2008;864:38-42.

88. Charles JJ, Geneste C, Kummer EL, Gheyouche R, Boudis H, Dubost JP. Development and validation of a rapid HPLC method for the determination of oseltamivir phosphate in Tamiflu and generic versions. J Pharma Biomed Anal 2007;44:1008-13.

89. Rajendran R, Devika S, Sura RS, Sunil V, Kumar MB, Gopinath P, et al. RP-HPLC pda method for estimation of zanamivir in api and pharmaceutical formulation. Indo Am J Pharm Sci 2018;5:2110-6.

90. Bhirud CH, Nandal DH. Stability indicating RP-HPLC and HPTLC methods for the determination of zanamivir in bulk and dosage form. Int J Pharm Sci 2016;8:249-56.

91. Reddy YR, Harika KSL, Sowjanya KS, Swathi E, Soujanya B, Reddy SS. Estimation of zanamivir drug present in tablets using RP-HPLC method. Int J PharmTech Res 2011;3:180-6.

92. Boonyapiwat B, Sarisuta N, Ma Y, Steventon GB. A validated HPLC method for zanamivir and its application to in vitro permeability study in caco-2 culture model. Indian J Pharm Sci 2011;73:564-8.

93. Erk N. A validated HPLC method for the determination of the neuraminidase inhibitor, zanamivir (gg167), in spiked human plasma and in pharmaceutical formulations. J Liq Chromatogr Related Tech 2004;27:1541-52.

94. Wang F, Zhang S, Sheng C, Zhao X, You J. Sensitive determination of amantadine in microdialysis samples from rat plasma by HPLC with fluorescence detection. J Liq Chromatogr Relat Technol 2015;38:1622-8.

95. Yanamadala G, Lavanya N, Srikumar PP. A pre-column derivatization technique for the development and validation of a HPLC-UV method for the determination of amantadine hydrochloride in bulk and formulations by using (2-napthoxy) acetyl chloride. Acta Chim Pharm Indica 2014;4:170-9.

96. Vemuri SK, Krishna NR, Mohammad MJ, Nalluri BN. Analysis of amantadine hydrochloride-phenyl isothiocyanate complex in bulk and pharmaceutical dosage forms by RP HPLC-PDA method. Br J Pharma Res 2014;4:278-88.

97. Darwish IA, Aboul FT, Khalil NY, Mahmoud AM, Al-Obaid AM. New sensitive hplc method for evaluation of the pharmacokinetics of new amantadine prodrugs as hepatic delivery systems to enhance its activity against HCV. Int J Res Pharm Sci 2010;1:151-7.

98. Yihua Z, Jianguo J, Xuejing H, Shiliang Z. Simultaneous determination of three components in pediatric paracetamol and amantadine hydrochloride granules using high performance liquid chromatography with gradient elution and dual wavelength detection. Chin J Chromatogr 2010;28:1005-8.

99. Shuangjin C, Fang F, Han L, Ming M. New method for highperformance liquid chromatographic determination of amantadine and its analogues in rat plasma. J Pharma Biomed Anal 2007;44:1100-5.
100. Higashi Y, Uemori I, Fujii Y. Simultaneous determination of amantadine and rimantadine by hplc in rat plasma with precolumn derivatization and fluorescence detection for pharmacokinetic studies. Biomed Chromatogr 2005;19:655-62.

101. Mamatha J, Devanna N. Development and validation of a rphplc method for the analysis of rimantadine hydrochloride in medicinal form. Rasayan J Chem 2018;11:300-6.

102. Zacharis CK, Tzanavaras PD, Vlessidis AG. Determination of rimantadine in human urine by hplc using a monolithic stationary phase and on-line post-column derivatization. J Sep Sci 2013;00:1-6.

103. Bhirud CH, Nandal DH. Development and validation of stability indicating hptlc method for the determination of oseltamivir phosphate in bulk and dosage form. Int J Pharm Pharma Res 2017;9:299-311.

104. Al Bratty M, Saleh SF, Alhazmi HA, Javed SA, Ahmed AM, Ahsan W. A validated hptlc densitometric method for quantitative determination of zanamivir in bulk and pharmaceutical formulation. Eur J Chem 2018;9:115-20.

105. Kummer EL, Gaudin K, Charles JJ, Gheyouche R, Boudis H, Dubost JP. Development and validation of a rapid capillary electrophoresis method for the determination of oseltamivir phosphate in Tamiflu and generic versions. J Pharma Biomed Anal 2009;50:544-6.

106. Pazourek J, Revilla AL, Gajdosova D, Havel J. Validation of a capillary zone electrophoresis method for determination of rimantadine hydrochloride in rimantadine 100 tablets and the method application to dissolution test monitoring. Drug Dev Ind Pharm 2004;30:125-34.

107. Revilla AL, Hamacek J, Lubal P, Havel J. Determination of rimantadine in pharmaceutical preparations by capillary zone electrophoresis with indirect detection or after derivatization. Chromatogr 1998;47:433-9.

108. Rashed NS, Abdallah OM, Said NS. Validated stability-indicating methods for determination of oseltamivir phosphate. $\mathrm{Br} \mathrm{J}$ Pharma Res 2017;16:1-9.

109. Mehta SD, Paliwal S. Phytochemical analysis, liquid chromatography, and mass spectroscopy and in vitro anticancer activity of annona squamosa seeds linn. Asian J Pharm Clin Res 2018;11:101-3.

110. Nagappan K, Yamjala K, Sathyaseelan M, Byran G. Stability evaluation of tartrazine by liquid chromatography-diode array detector and high-resolution electron spray ionization quadrupole time-of-flight mass spectrometry/mass spectrometry analysis. Asian J Pharm Clin Res 2017;10:295-9.

111. Virani P, Sojitra R, Raj H, Jain V. Chromatographic method for irbesartan and its combination with other drug. J Crit Rev 2015;2:7-11.

112. Pradeep PS, Shrungesh KTO, Prashantha N, Mahadevan KM. Synthesis, in vitro antibacterial, toxicity and molecular docking anticancer activity of novel n-[(2-chloroquinolin-3-yl) methylidene]-2-aniline Schiff bases. Int J Curr Pharm Res 2015; 7:37-46.

113. Yahdiana H, Norma A, Harmita. Method development and validation of lercanidipine in human plasma by liquid chromatography tandem-mass spectrometry. Int J Appl Pharm 2018;10:87-91.

114. Bhadoriya A, Rathnam S, Dasandi B, Parmar D, Sanyal M, Shrivastav PS. Sensitive and rapid determination of amantadine without derivatization in human plasma by LC-MS/MS for a bioequivalence study. J Pharma Anal 2018;8:202-7.

115. Wang K, Chen M, Weng H, Gao Y, Zhao H, Lin Z. Validation of a robust and high-throughput HPLC-MS/MS method to determine Amantadine levels in human plasma. J Appl Bioanal 2018;4:51-61.

116. Tsuruoka Y, Nakajima T, Kanda M, Hayashi H, Matsushima Y, Yoshikawa S, et al. Simultaneous determination of amantadine, rimantadine, and memantine in processed products, chicken tissues, and eggs by liquid chromatography with tandem mass spectrometry. J Chromatogr B 2017;1044-1045:142-8.

117. Turnipseed SB, Storey JM, Andersen WC, Filigenzi MS, Heise AS, Lohne JJ, et al. Determination and confirmation of the antiviral drug amantadine and its analogues in chicken jerky pet treats. J Agric Food Chem 2015;63:6968-78. 
118. Lindberg RH, Fedorova G, Blum KM, Pulit-Prociak J, Gillman A, Jarhult J, et al. Online solid phase extraction liquid chromatography using bonded zwitterionic stationary phases and tandem mass spectrometry for rapid environmental trace analysis of highly polar hydrophilic compounds-application for the antiviral drug Zanamivir. Talanta 2015;141:164-9.

119. Janiwarad S, Haq KU, Choudhary D. A selective and sensitive liquid chromatographic/tandem mass spectrometric method for simultaneous estimation of oseltamivir and its metabolite oseltamivir carboxylic acid in human plasma for bioavailability or bioequivalence studies. World J Pharma Res 2014;3:4598-614.

120. Zhao S, Li D, Qiu J, Wang M, Yang S, Chen D. Simultaneous determination of amantadine, rimantadine and chlorpheniramine in animal derived food by liquid chromatography-tandem mass spectrometry after fast sample preparation. Anal Methods 2014;6:7062-7.

121. Hu ZY, Laizure SC, Meibohm B, Herring VL, Parker RB. Simple and sensitive assay for quantification of oseltamivir and its active metabolite oseltamivir carboxylate in human plasma using high-performance liquid chromatography coupled with electrospray ionization tandem mass spectrometry: improved applicability to pharmacokinetic study. J Pharma Biomed Anal 2013;72:245-50.

122. Gupta A, Guttikar S, Shrivastav PS, Sanyal M. Simultaneous quantification of prodrug oseltamivir and its metabolite oseltamivir carboxylate in human plasma by LC-MS/MS to support a bioequivalence study. J Pharma Anal 2013;3:149-60.

123. Lin CC, Yen JC, Wu YT, Lin LC, Tsai TH. Chemical analysis and transplacental transfer of oseltamivir and oseltamivir carboxylic acid in pregnant rats. PLoS One 2012;7:e46062.

124. Berendsen BJA, Wegh RS, Essers ML, Stolker AAM, Weigel S. Quantitative trace analysis of a broad range of antiviral drugs in poultry muscle using column-switch liquid chromatography coupled to tandem mass spectrometry. Anal Bioanal Chem 2012;402:1611-23.

125. Kromdijk W, Rosing H, Van den Broek MPH, Beijnen JH, Huitema ADR. Quantitative determination of oseltamivir and oseltamivir carboxylate in human fluoride EDTA plasma including the ex vivo stability using high-performance liquid chromatography coupled with electrospray ionization tandem mass spectrometry. J Chromatogr B 2012;891-892:57-63.

126. Lindegardh N, Hanpithakpong W, Kamanikom B, Farrar J, Hien TT, Singhasivanon P, et al. Quantification of the anti-influenza drug zanamivir in plasma using high-throughput HILICMS/MS. Bioanal 2011;3:157-65.
127. Kanneti R, Bhavesh D, Paramar D, Shivaprakash R, Bhatt PA. Development and validation of a high-throughput and robust LCMS/MS with electrospray ionization method for simultaneous quantitation of oseltamivir phosphate and its oseltamivir carboxylate metabolite in human plasma for pharmacokinetic studies. Biomed Chromatogr 2011;25:727-33.

128. Baughman TM, Wright WL, Hutton KA. Determination of zanamivir in rat and monkey plasma by positive ion hydrophilic interaction chromatography (HILIC)/tandem mass spectrometry. J Chromatogr B 2007;852:505-11.

129. Arndt T, Guessregen B, Hohl A, Reis J. Determination of serum amantadine by liquid chromatography-tandem mass spectrometry. Clin Chim Acta 2005;359:125-31.

130. Jia Q, Li D, Wang X, Yang S, Qian Y, Qiu J. Simultaneous determination of amantadine and rimantadine in feed by liquid chromatography-Qtrap mass spectrometry with informationdependent acquisition. Anal Bioanal Chem 2018;410:5555-65.

131. Mu P, Xu N, Chai T, Jia Q, Yin Z, Yang S, et al. Simultaneous determination of 14 antiviral drugs and relevant metabolites in chicken muscle by UPLC-MS/MS after QuEChERS preparation. J Chromatogr B 2016;1023-1024:17-23.

132. Azuma T, Ishiuchi $H$, Inoyama T, Teranishi $Y$, Yamaoka M, Sato $\mathrm{T}$, et al. Detection of peramivir and laninamivir, new antiinfluenza drugs, in sewage effluent and river waters in japan. PloS One 2015;10:e0131412.

133. Takanami R, Ozaki H, Giri RR, Taniguchi S, Hayashi S. Antiviral drugs zanamivir and oseltamivir found in wastewater and surface water in Osaka, Japan. J Water Environ Tech 2012;10:57-68.

134. Takanami R, Ozaki H, Giri RR, Taniguchi S, Hayashi S. Detection of antiviral drugs oseltamivir phosphate and oseltamivir carboxylate in neya river, Osaka, Japan. J Water Environ Tech 2010;8:363-72.

135. Yan H, Liu X, Cui F, Yun H, Li J, Ding S, et al. Determination of amantadine and rimantadine in chicken muscle by QuEChERS pretreatment method and UHPLC coupled with LTQ Orbit rap mass spectrometry. J Chromatogr B 2013;938:8-13.

136. Wu YL, Chen RX, Xue Y, Yang T, Zhao J, Zhu Y. Simultaneous determination of amantadine, rimantadine and memantine in chicken muscle using multi-walled carbon nanotubes as a reversed-dispersive solid phase extraction sorbent. J Chromatogr B 2014;965:197-205.

137. Hooff GP, Meesters RJW, Van Kampen JJA, Van Huizen NA, Koch $\mathrm{B}$, Al Hadithy AFY, et al. Dried blood spot UHPLC-MS/MS analysis of oseltamivir and oseltamivir carboxylate-a validated assay for the clinic. Anal Bioanal Chem 2011;400:3473-79. 